\title{
Polysémie régulière: questions de représentation
}

\author{
Achim Stein* \\ Universität Stuttgart, Institut für Linguistik/Romanistik \\ achim.stein@ling.uni-stuttgart.de
}

\begin{abstract}
Le propos de ce point de vue se situe entre les deux extrèmes de l'énumération des sens d'une part et de la schématisation abstraite de l'autre. Sans être génératif dans le sens du lexique génératif de Pustejovsky, il abordera les questions du transfert de sens, de la représentation sémantique et de la désambiguïsation automatique des verbes polysémiques.
\end{abstract}

\section{Introduction}

Sur le plan théorique, notre propos est un enrichissement de Stein (2005), où nous avons proposé un système conceptuel hiérarchique qui représentait les sens des verbes polysémiques à travers des liens entre les sens (unités du lexique) et les concepts (nœuds de la hiérarchie conceptuelle). Dans Stein (2007) nous avons élaboré cette aproche en proposant une représentation sémantique non relationnelle (comme dans WordNet), mais décompositionnelle. A présent, nous essayons d'utiliser des outils ontologiques nous permettant de représenter formellement ces concepts et de les intégrer dans des applications.

Nous allons illustrer notre propos en présentant l'analyse du verbe pousser et le transfert de sens du domaine physique et physique (marqué par l'index $\phi$ ) au domaine psychologique (marqué par l'index $\psi$ ).

\footnotetext{
${ }^{*}$ Ce propos présente les résultats élaborés en collaboration avec Fabienne Martin et Dennis Spohr dans le projet B5 «Polysemy in a Contextual System», qui fait parti du SFB 732 Incremental Specification in Context, Université de Stuttgart.
} 


\section{Analyse sémantique}

\section{1 pousser physique}

Dans l'analyse de pousser (Stein, 2007), la comparaison des représentations du concept PUSH dans plusieurs systèmes conceptuels (WordNet, FrameNet, Mikrokosmos) a révélé des insuffisances dues surtout à l'absence d'un mécanisme voir d'une théorie qui aurait pu relier les sens entre eux. En nous appuyant sur les concepts d'identité et d'unité définis par Guarino et Welty, nous avons tenté d'identifier ces propriétés dans le contenu référentiel de ce verbe.

Strictly speaking, identity is related to the problem of distinguishing a specific instance of a certain class from other instances by means of a characteristic property, which is unique for it (that whole instance). Unity, on the other hand, is related to the problem of distinguishing the parts of an instance from the rest of the world by means of a unifying relation that binds them together (not involving anything else). Guarino \& Welty (2001)

Cette analyse a confirmé le classement de pousser parmi les verbes dénotant l'exercice d'une force. Les exemples suivants montrent en effet, que le mouvement de l'agent $x$ est un composant essentiel de la situation exprimée par pousser, puisque la contradiction de ce composant produit un contresens (1), et les adverbes modifiant ce composant sont acceptables (2).

(1) \# Il a poussé la boîte sans bouger.

(2) Il a poussé la boîte énergiquement (mais elle n'a pas bougé).

Par contre, l'agent $x$ ne doit pas forcément changer de place (3), et le déplacement du patient $y$ n'est pas impliqué non plus (4) :

(3) Assis dans son fauteuil, il a poussé la boîte dans un coin.

(4) Max a poussé la table (mais elle n'a pas bougé).

Pour Jackendoff (1990, 90 et 133) push décrit une causation continue du mouvement qui, contrairement aux causatifs pures comme rompre, n'aboutit pas à un résultat déterminé - analyse d'ailleurs confirmée par le Petit Robert : «Soumettre (qqch., qqn) à une force agissant par pression ou par choc et permettant de mettre en mouvement, et de déplacer dans une direction.» (Petit Robert, 1993).

pousser n'est pas polysémique, mais vague quant aux relations temporelles qui relient les sous-événements, notamment par rapport à la durée du contact (essentiel), qui peut 
dans une interprétation maximale avoir commencé avant le mouvement de l'agent $x$ et continuer après la fin du déplacement du patient $y$, et se limiter dans une interprétation minimale au moment de transition entre les deux mouvements. L'application du test de Lakoff (1970) dans (5) montre que les deux interprétations ne sont pas incompatibles :

(5) Max a prudemment poussé le carton dans un coin, et la chaise avec son pied dans l'autre.

Pour résumer les relations temporelles entre les sous-événements, nous utilisons $<_{\propto}$ 'exhaustive ordered part', $\circ_{\propto}$ 'exhaustive overlap part of' (pour les sous-événements simultanés), $<o_{\infty}$ 'exhaustive ordered overlap' (cf. Kamp $(1979,139)$; ainsi que Pustejovsky (1995, 69-71), qui exemplifie les réalisations verbales de ces réalisation par les verbes build, accompany et walk, respectivement.) Les index indiquent le mouvement $m$ de l'agent, le contact $c$ et le déplacement $d$ du patient.

(6) $\quad\left(\mathrm{e}_{m}<\mathrm{e}_{c}\right) \wedge\left(\mathrm{e}_{c}<\mathrm{e}_{d}\right)$ ['onset causation', cf. Talmy 2000, 473]

(7) $\left(\mathrm{e}_{m}<\mathrm{o}_{\propto} \mathrm{e}_{c}\right) \wedge\left(\mathrm{e}_{c}<\circ_{\propto} \mathrm{e}_{d}\right)$ ['mouvement partiellement accompagné']

(8) $\left(\mathrm{e}_{m}<\circ_{\propto} \mathrm{e}_{c}\right) \wedge\left(\mathrm{e}_{c} \circ_{\propto} \mathrm{e}_{d}\right)$ ['mouvement accompagné']

Dans (6), le mouvement de l'agent $x$ precède le contact, et le contact précède le déplacement sans chevauchement : le contact est donc le moment de transition entre les deux mouvements (ce qui correspond à la définition pousser:11 'flick' de WordNet). (7) et (8) représentent la plupart des autres sens subsumés par pousser : 6 et sont vagues par rapport à la relation entre le contact et le déplacement, car le contact peut cesser ou non avant la fin du déplacement.

\section{2 pousser psychologique}

Le déplacement du patient $y$ n'est donc pas une propriété essentielle de ousser $_{\phi}$. En termes ontologiques, nous pourrions parler d'une propriété «non rigide» de la situation physique : d'après Guarino \& Welty (2001), une propriété essentielle ${ }_{\phi}$ est vrai pour un individu $a$ à tout moment possible et dans tous les mondes possibles $(\square \forall t \phi(a, t))$.

L'application de cette analyse au sens psychique, pousser $_{\psi}$, se fait sans problème, puisque le «contact» (verbal ou social) précède l'action accomplie par le patient $y$, dans (9) :

(9) Son copin l'a poussé à cambrioler le magasin.

F. Martin approfondit l'analyse du domaine psychologique (Martin, 2008). Elle constate d'une part que la présence d'une phrase infinitive introduite par à interdit l'interprétation 
physique, et de l'autre qu'il est difficile d'arriver à une interprétation psychologique si le complément d'objet direct est absent. Cette sensibilité de pousser vis-à-vis de son entourage syntaxique semble confirmer l'hypothèse que c'est surtout la réalisation des arguments qui produit les effets de sens polysémiques et détermine le sens du prédicat dans le contexte. (cf. Koch 1991). Il est vrai que certains types de compléments désambiguïsent le verbe : en principe, la préposition à peut introduire la direction ou la cible du déplacement de $y$, pourtant elle est rare avec les compléments locaux de pousser $_{\phi}$, mais caractéristique de pousser $_{\psi}$.

(10) L'angoisse me poussa à sa rencontre/à le rencontrer.

Quant à $\operatorname{pousser}_{\phi}$, F. Martin constate que certains types de compléments locaux impliquent que le déplacement de y a eu lieu, comme dans (11), contrairement à (12). Elle parle alors de verbes «implicatifs» (dans le contexte des verbes psychologiques, cf. Martin 2006, 393) :

(11) \# Il a poussé la boîte dans la salle de bains/jusqu'au mur, mais elle n'a pas bougé.

(12) Il a poussé la boîte vers le mur, mais elle n'a pas bougé.

\subsection{Conditions de transfert sémantique}

En spécifiant les conditions de transfert sémantique, nous allons essayer de démontrer que ce n'est pas le changement de la structure argumentale qui coerce le sens du verbe. C'est la structure sémantique du verbe qui définit son potentiel polysémique, et cette structure, au lieu d'être abstraite ou schématique doit être au contraire suffisamment concrète et détaillée, pour éviter une «surgénération» de sens. Nous allons donc considérer de plus près les propriétés de pousser $_{\phi}$ dont hérite pousser $_{\psi}$.

Une des ces propriétés se montre dans le fait que le projet $w$ exprimé par le complément prépositionnel — tout comme le déplacement de $y$ de pousser $_{\phi}$ — ne doit pas avoir commencé, cf. (14) :

(13) Moi ce qui m'encourage à vivre c'est la musique.

(14) 'Moi ce qui me pousse à vivre c'est la musique.

Ensuite, pousser $_{\psi}$ se distingue des verbes psychologiques qui n'ont pas de sens physique, comme encourager dans (15), par l'héritage du complément locatif ou directionnel :

(15) *Je l'ai encouragé vers le catholicisme. 
(16) Je l'ai poussé vers le catholicisme.

(17) Tu m'as poussé jusqu' au meurtre de cette minable racaille.

Dans ce contexte, F. Martin évoque la question de savoir si la direction ou l'état final est conçu (et devrait donc être représenté) comme une place abstraite.

Enfin, pour conclure la discussion des analogies entre pousser $_{\phi}$ et pousser $_{\psi}$, il s'agira de concrétiser la notion de contact qui, d'après notre analyse, était une propriété essentielle de pousser $_{\phi}$. Il semblerait en effet que pour expliquer les types de compléments locatifs (pousser contre le mur etc.) par lesquels pousser s'apparente plus d'appuyer que de toucher, il faille aller plus loin et introduire la notion de résistance, ce qui fait d'ailleurs F. Martin (2008) en postulant un obstacle présupposé par pousser $_{\phi}$ qui rend l'expression d'un résultat difficile (18) et qui différencie pousser, dans les deux domaines, des verbes qui sont neutres par rapport à cette propriété, comme toucher (19) ou vouloir (21).

(18) Les roses ont (\#bien) poussé des racines (\# de deux centimètres)

(19) Il touche (*contre) la table.

(20) Il pousse (contre) la table.

(21) Je veux partir, et lui aussi veut que je parte.

(22) Je veux partir, \# et lui aussi me pousse à partir.

Dans la partie 3, nous essayons de formuler une représentation qui tienne compte des propriétés retenues ici.

\section{Représentation et traitement}

\subsection{Questions de représentation}

Nous utilisons une représentation néo-davidsonienne, car elle permet de formuler les règles SWRL (cf. infra) assez facilement.

La représentation de pousser $_{\phi}$ (23) inclut la résistance comme une force antagoniste dont le vecteur est opposé à celui de la force exercée par l'agent $x$ qui agit sur $y$ en direction de $z$, pourvu qu'il y ait déplacement). ${ }^{1}$ Le vecteur de la force antagoniste est donc spécifié par rapport à sa direction (l'arrivé est le lieu de contact $c$ entre $x$ et $y$ ) et sous-spécifié par rapport à son point de départ (situé quelque part entre $z$ et $c$ ). Ceci est représenté dans (23) pour pousser $_{\phi}$.

\footnotetext{
${ }^{1}$ Nous utilisons «antagoniste» dans le sens courant du mot, 'opposé', bien que Talmy attribue le rôle d'AGONISTE à l'objet sujet et celui d'ANTAGONISTE au sujet de pousser.
} 


$$
\begin{aligned}
& \text { pousser }_{\phi} \Rightarrow \lambda v \lambda m \lambda z \lambda y \lambda x \lambda e[\operatorname{Agent}(x, e) \wedge \operatorname{Target}(y, e) \wedge \operatorname{Goal}(z, e) \wedge \\
& \operatorname{Force}(m, v) \wedge v(\text { orig, }, \wedge) \wedge x \leq \operatorname{orig} \leq c \\
& \left.\rightarrow P \exists \operatorname{Force}^{\prime}\left(m^{\prime}, v^{\prime}\right) \wedge v^{\prime}\left(\text { orig }^{\prime}, c\right) \wedge z \leq \operatorname{orig}^{\prime} \leq c \wedge m^{\prime} \leq m\right]
\end{aligned}
$$

La représentation de pousser $\psi$ peut être égale à celle de (23) si on souscrit à une vue métaphorique de la dynamique des forces à la Talmy. Le vecteur $v$ correspondrait alors, dans le domaine psychique, à la volonté de $x$ par rapport à une proposition $\mathrm{P}$, et le vecteur $v^{\prime}$ à la volonté opposée (non-volonté) de $y$ par rapport à cette même proposition. Cette représentation exprimerait directement le lien entre les acceptions de pousser. D'autre part, pousser $_{\psi}$ est sémantiquement proche de certains autres verbes comme inciter ou encourager qui eux n'ont pas de sens physique et qui expriment le changement du degré $d$ de volonté par rapport à $\mathrm{P}$ à travers d'un prédicat $\operatorname{Want}(s, d, P, t)$. Dans le cas de pousser $\psi$, nous traduirions le vecteur opposé $v^{\prime}$ par un degré de volonté négatif $d<0$.

\subsection{Désambiguïsation}

Identifier les inférences qu'on peut tirer à partir de l'occurrence d'un verbe dans le contexte est crucial pour la compréhension d'un texte et pour des applications comme le text mining ou le résumé automatique. La description sémantique présentée dans la partie 2 est traitée dans un système qui la modélise en utilisant OWL DL et SWRL ${ }^{2}$. Nous n'en pouvons fournir ici qu'une description sommaire. ${ }^{3}$

Dans ce système, nous représentons les restrictions de sélection traditionnelles par la présence et le type ontologique des arguments en nous fondant sur l'ontologie SUMO ${ }^{4}$. Les différents sens d'un verbe sont des sous-classes d'une représentation sous-spécifiée. Ces sens ne sont pas seulement caractérisés par les restrictions argumentales, mais aussi par les inférences qu'ils permettent de tirer. Ces inférences sont codées par des règles SWRL : Ces règles spécifient

1. la configuration syntaxique contenant la structure argumentale

2. la création des différents prédicats de la représentation formelle (règles swrlx: $\operatorname{coT}(\ldots))$ : degrés, unités temporelles etc.

3. l'assertion des différents prédicats : rôles sémantiques, propositions

Dans notre système conceptuel, nous essayons de généraliser ces représentations en réutilisant par exemple des ensembles de règles qui sont partagés par un sous-domaine conceptuel : ainsi, les concepts dénotés par pousser partagent une partie des règles avec

\footnotetext{
${ }^{2}$ OWL : Web Ontology Language, DL : Description Logic, SWRL : Semantic Web Rule Language

${ }^{3}$ Pous plus de détails cf. Martin et al. (2008).

${ }^{4}$ Suggested Upper Merged Ontology (Niles \& Pease, 2003)
} 
des verbes comme inviter, inciter, encourager etc. Ces règles deviennent alors des valeurs de propriétés de certaines classes conceptuelles.

Dans le procès de désambiguïsation, le système traite d'abord les ambiguïtés des arguments du verbe (identifiés par un parseur) en utilisant les équivalences entres le WordNet français et SUMO. Les classes de ces arguments sont alors insérées dans une instance du concept qui représente le verbe (par exemple, dans le cas de pousser $\psi$, les arguments $x$ et $y$ seraient remplacés par une classe PERSONNE). Le système applique alors les règles SWRL pour déterminer la sens qui correspond le mieux aux contraintes imposées par la structure argumentale. Nous pouvons alors calculer les inférences en combinant les règles SWRL avec la Jess rule engine ${ }^{5}$ qui nous fournit par exemple les informations contextuelles sur les différents arguments (' $x$ a bougé', ' $y$ s'est déplacé', 'le degré de volonté de $y$ a changé' etc.).

Nous utilisons l'éditeur ontologique Protégé $e^{6}$ pour définir les classes de concepts ainsi que les règles SWRL, avec les plugins respectifs.

\section{Résumé de notre point de vue}

Nous avons proposé de représenter la polysémie verbale en combinant une hiérarchie conceptuelle ( «ontologie») avec une description sémantique formelle, dans le but

- de représenter l'interaction entre le contexte syntaxique et la structure sémantique du verbe,

- de modéliser la description linguistique en utilisant des formalismes ontologiques (OWL, SWRL),

- d'implémenter un système de désambiguïsation fondé sur les inférences que la description sémantique permet de tirer.

\section{Références}

Guarino, N. \& Welty, C. (2001). Identity and Subsumption. - Green, R. \& Bean, C. \& Hyon Myaeng, S., eds., The Semantics of Relationships : An Interdisciplinary Perspective. Kluwer Academic Publishers, Dordrecht etc., 111-126.

Jackendoff, R. (1990). Semantic Structures. MIT Press, Cambrige Mass.

Kamp, H. (1979). Some Remarks on the Logic of Change, Part I. — Rohrer, C., ed., Time, Tense, and Quantifiers. Niemeyer, Tübingen, 135-179.

\footnotetext{
5 http: //www. jessrules.com

${ }^{6}$ http: //protege.stanford.edu
} 
Koch, P. (1991). Semantische Valenz, Polysemie und Bedeutungswandel bei romanischen Verben. - Koch, P. \& Krefeld, T., eds., Connexiones Romanicae. Dependenz und Valenz in den romanischen Sprachen. Niemeyer, Tübingen, 279-306.

Lakoff, G. (1970). A note on vagueness and ambiguity. Linguistic Inquiry, 1 :357-359.

Martin, F. (2006). Prédicats statifs, causatifs et résultatifs en discours. Sémantique des adjectifs évaluatifs et des verbes psychologiques. Université libre de Bruxelles, Bruxelles.

Martin, F. (2008). Forceful verbs in spatial, psychological and conceptual domains. Semantic analysis of 'push' verbs in French. unpublished ms.

Martin, F. \& Spohr, D. \& Stein, A. (2008). Disambiguation of Polysemous Verbs for Rule-based Inferencing. submitted to Computational Linguistics (march 2008).

Niles, I. \& Pease, A. (2003). Linking Lexicons and Ontologies : Mapping WordNet to the Suggested Upper Merged Ontology. - Proceedings of the 2003 International Conference on Information and Knowledge Engineering (IDE '03), Las Vegas, $N V$.

Petit Robert (1993). Le Nouveau Petit Robert. Dictionnaire alphabétique et analogique de la langue française, volume Nouvelle édition remaniée et amplifiée sous la rédaction de Josette Rey-Debove et Alain Rey. Le Robert, Paris.

Pustejovsky, J. (1995). The Generative Lexicon. A Theory of Computational Lexical Semantics. MIT Press, Cambrige Mass.

Stein, A. (2005). Semantische Repräsentation italienischer Verben. Automatische Disambiguierung mit Konzepthierarchien. Niemeyer, Tübingen. Linguistische Arbeiten ; 499.

Stein, A. (2007). Motion events in conceptual taxonomies : General considerations and French examples. - Zaefferer, D. \& Schalley, A., eds., Ontolinguistics. How ontological status shapes the linguistic coding of concepts. Mouton de Gruyter, Berlin, New York, 379-394, Trends in Linguistics.

Talmy, L. (2000). Toward a Cognitive Semantics. MIT Press, Cambridge. 